doi $10.22199 /$ issn. $2452-5774-4607$ SOCLAL INNOVATION STUDIES

ISSN: 2452-5774 (On line)

\title{
Formación de líderes sociales para el desarrollo local: una aproximación desde la innovación social
}

\section{Educating social leaders for local development: an approach from social innovation}

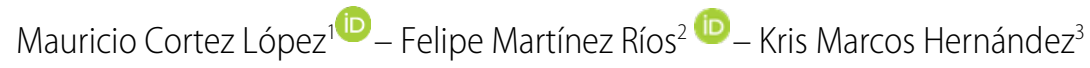 \\ 1,2,3. Plataforma NISA Consultores, Santiago, Chile. \\ @mcl.mauricio@gmail.com felipemartinezrios@gmail.com krisita.marcos@gmail.com
}

\section{(ㄷ) (1)}

Resumen:

Se presenta una propuesta de intervención social interdisciplinaria desde el enfoque de la innovación social, a través de la experiencia formativa desarrollada en una escuela de dirigentes sociales, en la comuna de Santiago (Chile). Se parte de una aproximación teórico-metodológica que busca ir más allá de la mirada de la innovación social centrada en mecanismos de mercado, a partir de la articulación de dos metodologías: el aprendizaje basado en proyectos y el design thinking. Se describe cómo se ha aplicado este enfoque en la escuela y se concluye que desarrollar competencias en innovación social en líderes sociales, bajo la metodología planteada, se puede vincular de manera directa con procesos de desarrollo local.

Palabras clave: intervención social interdisciplinaria, aprendizaje basado en proyectos, design thinking.

Abstract:

An interdisciplinary social intervention proposal from the social innovation approach is presented through the formative experience developed in a school of social leaders, in the commune of Santiago (Chile). The article entails a theoretical-methodological approach that goes beyond the perspective of social innovation focused on market mechanisms, from articulation of two methodologies: projectbased learning and design thinking. It is described how this approach has been applied at the school of social leaders, and it is concluded that developing competencies in social innovation in social leaders, under the proposed methodology, can be directly linked to local development processes.

Keywords: interdisciplinary social intervention; learning based on projects; design thinking. 


\section{Introducción}

La innovación social es tanto un enfoque teórico como una metodología de trabajo que ha ido ganando espacio como perspectiva de intervención social contemporánea (Espiau, 2017; Gatica, 2016; Rodríguez y Alvarado, 2008). A pesar de su amplia variedad de definiciones y aproximaciones, ha alcanzado ciertos niveles de autonomía y diferenciación con respecto a otras formas de intervención, lo cual se ha nutrido de decenas de experiencias e iniciativas que han surgido en la academia, el sector privado, el Estado y la sociedad civil, generando un panorama complejo de propuestas, muchas veces híbridas. La innovación social como eje de procesos de intervención social a escala local, a pesar de los avances, es aún un enfoque poco desarrollado en Latinoamérica y Chile, en donde han predominado lógicas de intervención focalizadas, tradicionales y sectoriales (Ministerio de Desarrollo Social, 2018), de forma que no ha logrado permear de manera relevante el statu quo en materia de intervención.

Para avanzar en el desarrollo de una propuesta de intervención social interdisciplinaria basada en la innovación social, es importante analizar casos empíricos que permitan sostener un diálogo teórico-metodológico entre los diversos actores interesados en estas temáticas. Este artículo propone describir reflexiva y críticamente un enfoque teórico-metodológico de intervención en torno a la formación de líderes sociales de la comuna de Santiago, denominada Escuela de Dirigentes Sociales. El diseño y ejecución de esta escuela fue desarrollado por los autores del artículo, por lo cual se puede inscribir dentro del paradigma de la investigación-acción.

El proceso formativo de líderes sociales, desde la perspectiva de la innovación social, implica no solo potenciar habilidades y conocimientos usualmente utilizados en esta área, tales como el codiseño, la creatividad, la elaboración de proyectos en equipo y el trabajo en red, sino también vincular estos elementos a los problemas y potencialidades de territorios específicos. Para alcanzar este objetivo, se plantea un enfoque de formación a través del aprendizaje basado en la elaboración de proyectos de innovación social (ABP-NISA), con la capacidad de gatillar procesos sinérgicos de innovación a escala local, al poner en valor y dinamizar un conjunto de elementos endógenos-en-red o reticulares (Monnet, 2013). Para ello, es fundamental que los líderes sociales desarrollen competencias en este paradigma, diferenciándose de los dirigentes tradicionales y del emprendedor -socialindividual (Mazzucato, 2014). Del mismo modo, es relevante que el impulso alcanzado por los líderes sociales a través de la Escuela de Dirigentes Sociales, se logre articular con instancias o modelos de planificación territorial cuyo foco esté puesto en el desarrollo local, tales como el enfoque triple hélice (Etzkowitz y Leydesdorff, 1995) y multihélice (Concha y Ricci, 2018), el modelo de valor compartido (Porter y Kramer, 2011), o proyectos de gobiernos locales asociados a planes comunales de desarrollo, por mencionar algunos.

El artículo se compone de tres partes. En la primera, se formula un marco teórico que vincula modelos de desarrollo con procesos de innovación social, con el fin de clarificar la posición teórica- 
metodológica que se propone. A continuación, se identifican elementos claves de un enfoque de intervención interdisciplinaria centrada en la formación de líderes sociales desde el paradigma de la innovación social, señalando las definiciones y articulaciones más importantes. En la segunda parte, se caracteriza el caso de la Escuela de Dirigentes Sociales con la finalidad de ilustrar la aplicación del enfoque, así como el proceso de adaptación realizado por el equipo de NISA Consultores. Finalmente, el análisis integrado de estas dos partes permite visualizar de manera sintética un proceso completo de intervención que transita desde la formación de líderes hasta el desarrollo local.

\section{Metodología}

La metodología empleada en la elaboración de este artículo se inscribe en el enfoque denominado investigación - acción, aplicada desde el aprendizaje basado en proyectos (Botella y Ramos, 2018), lo cual consiste en estudiar e intervenir en la realidad de manera simultánea durante el proceso investigativo, generando conocimiento a partir de la práctica de interacción con las personas. En este caso, a partir del proceso de formación en la Escuela de Dirigentes Sociales de Santiago. La investigación-acción debe acercarse a la realidad para comprenderla y cambiarla, transformando la práctica educativa (Colmenares, 2012). En términos del enfoque cualitativo empleado, se trata, entonces, de un estudio de caso único (Gerring, 2016), el cual permite profundizar en sus distintas dimensiones con la finalidad de establecer futuras comparaciones entre casos de " $\mathrm{N}$ " mayor. De este modo, esta aproximación contribuye a la generación de teoría específica sobre innovación social.

Este estudio implicó, entonces, dos ejes principales de trabajo: Por una parte, la revisión de literatura especializada sobre innovación social con el objetivo de diseñar un enfoque teóricometodológico de intervención relativamente propio. Para ello se realizó un análisis crítico del estado del arte de la innovación social, con el objetivo de presentar una mirada equilibrada e integradora como propuesta de intervención. En el segundo eje, empírico, se analizó el conjunto de experiencias a partir del desarrollo de la Escuela de Dirigentes Sociales en tres de sus versiones ocurridas entre los años 2018 y 2019. Esto implicó analizar los resultados del proceso formativo implementado con 162 dirigentes sociales, a partir de lo cual se seleccionaron cuatro prototipos de innovación social que ilustran dichos resultados. En este sentido, la selección de prototipos consideró los siguientes criterios: (a) Haber sido co-diseñado y construido en su totalidad por dirigentes sociales; (b) incorporar todas las etapas del aprendizaje basado en proyectos y la metodología design thinking; (c) tener como finalidad de intervención el desarrollo local; y, finalmente, (d) tener el potencial de ser replicado a escala local en otros territorios. 


\section{Diseño teórico-metodológico: Innovación social para el desarrollo local Innovación social y desarrollo}

Las intervenciones sociales que plantean entre sus ejes a la innovación social se pueden agrupar en, al menos, tres enfoques, según el modelo de desarrollo al que adscriben, ya sea de manera declarada o tácita. Una de ellas se enmarca en el modelo de desarrollo liberal, el cual ha evolucionado desde centrarse en el crecimiento económico a secas, hasta la incorporación de elementos subjetivos y de bienestar humano y social (Malik, 2013). Podría decirse que es la visión con mayor presencia pública en innovación social. Los planteamientos de Yunus (2006) sobre los créditos a sectores tradicionalmente excluidos de la población -conocido como el Banco Grameen o "banco de los pobres"-, o de Prahalad (2005) en torno a su publicación La Riqueza en la Base de la Pirámide, sostienen que es posible integrar comercialmente a sectores de la población actualmente excluidos. En términos metodológicos, estas intervenciones sociales incorporan a las personas participativamente, mejorando su acceso a bienes y servicios de los que estaban privados desde la mirada del mercado tradicional. Este conjunto de iniciativas busca crear puentes entre comunidades, ONG, Estados y empresas privadas a través de interesantes modelos híbridos, cercanos a lo que Porter y Kramer (2011) denominaron "valor compartido", poniendo especial énfasis en la idea de impacto al articular iniciativas locales con necesidades globales. En este enfoque, los mecanismos de mercado son centrales en el desarrollo de la innovación social.

Al otro extremo se sitúa una mirada alternativa al actual modelo de desarrollo, que busca generar un tránsito entre nuestro modo de vivir actual y el futuro (Manzini, 2015). Esta aproximación ve en la innovación social una forma de co-diseñar este nuevo mundo, el cual debería superar la crisis socio-ambiental en la que vivimos y orientarnos hacia un nuevo modelo civilizatorio, más equilibrado, justo y plural (Escobar, 2017). Enfoques asociados al postdesarrollo o al postextractivismo (Svampa, 2016), por mencionar algunas aproximaciones relevantes en Latinoamérica, podrían ver en la innovación social un mecanismo de diseño participativo que facilite la creación de los modelos cognitivos, prácticas, valores e instituciones que lleven al mundo hacia una nueva forma de vivir (Escobar, 2017; Manzini, 2015). En este paradigma, las iniciativas son fuertemente transformadoras y rebeldes frente al statu quo actual. Destacan aquí iniciativas tales como los bancos de semilla, que persiguen la soberanía alimentaria frente a la propiedad de las empresas transnacionales, o experiencias eco-feministas, las cuales serían indispensables para la construcción de un "buen vivir" no capitalista ni patriarcal. Es interesante señalar que el término innovación social no es muy utilizado en este paradigma de transformación, sin embargo, dadas sus características, sus iniciativas pueden ser consideradas como innovaciones.

Entre uno y otro modelo se encuentran las perspectivas que, utilizando algunas herramientas de mercado y considerando el contexto de crisis socio-ecológica global, se enfocan en la dimensión territorial del mismo, siguiendo la línea del desarrollo local (Albuquerque, 2004; Boisier, 2010). Esta 
mirada busca identificar, poner en valor y fortalecer a un conjunto de elementos endógenos del territorio y de las comunidades que los habitan, siendo la creatividad, la cooperación y la confianza algunos de sus componentes centrales. Se pone énfasis en los procesos de diálogo entre los distintos actores a nivel territorial (Boisier, 2010). Experiencias destacadas en este sentido son las planteadas por el catastro y los concursos de innovación social realizados por la CEPAL (Rodríguez y Alvarado, 2008), algunas actividades realizadas por la Fundación para la Superación de la Pobreza, y el Modelo Multihélice de Innovación Social de la Universidad Católica del Norte (Concha y Ricci, 2018).

Desde esta última perspectiva, entendemos que la innovación social surge de procesos de diálogo y cooperación entre actores locales que buscan soluciones creativas a problemas socioecológicos que no han tenido una respuesta oportuna. En este marco, una de sus definiciones tradicionales señala que la innovación social se refiere a la mejora de alguna dimensión la vida de las personas, a través del uso y re-significación de un conjunto de elementos disponibles en la cultura (objetos, espacios, relaciones, entre otros), poniendo el acento en la creatividad social más que en un potencial y aislado invento (Cahill, 2010). Esta aproximación conceptual a la innovación social requiere situar espacialmente los recursos que se utilizan en los procesos estratégicos de innovación, por lo cual las intervenciones requieren un enfoque territorial. Del mismo modo, esta mirada se nutre y apunta a fortalecer procesos de acción colectiva por sobre individualidades, así como perspectivas multidimensionales en lugar de focalizadas, reduccionistas y sectoriales.

\section{Enfoque de innovación social territorial}

La innovación social muchas veces se instala en espacios donde ni el Estado ni el mercado han logrado llegar (Hernández et al., 2016), y en donde la innovación tradicional -industrial, tecnológica y de mercado- no tiene suficiente interés. En este marco, la innovación social se asocia a procesos de cohesión social, participación y desarrollo territorial, entre otros (Moulaert et al., 2010), y busca generar estrategias de cambio y transformación de amplio alcance (Córdoba-Cely et al., 2014), lo que incorpora una mirada de largo plazo, articulada a los procesos de gobernanza local. En este proceso de articulación y diálogo, se ha transitado desde la figura del emprendedor como principal agente de innovación social, a una amplia articulación de actores que sirven de soporte a las transformaciones sociales a nivel local (Hernández et al., 2016). Siguiendo esta ruta, se identifican ciertos conceptos fundamentales para la implementación de intervenciones sociales interdisciplinarias con foco en el desarrollo local, los cuales se esquematizan en la Figura 1. 


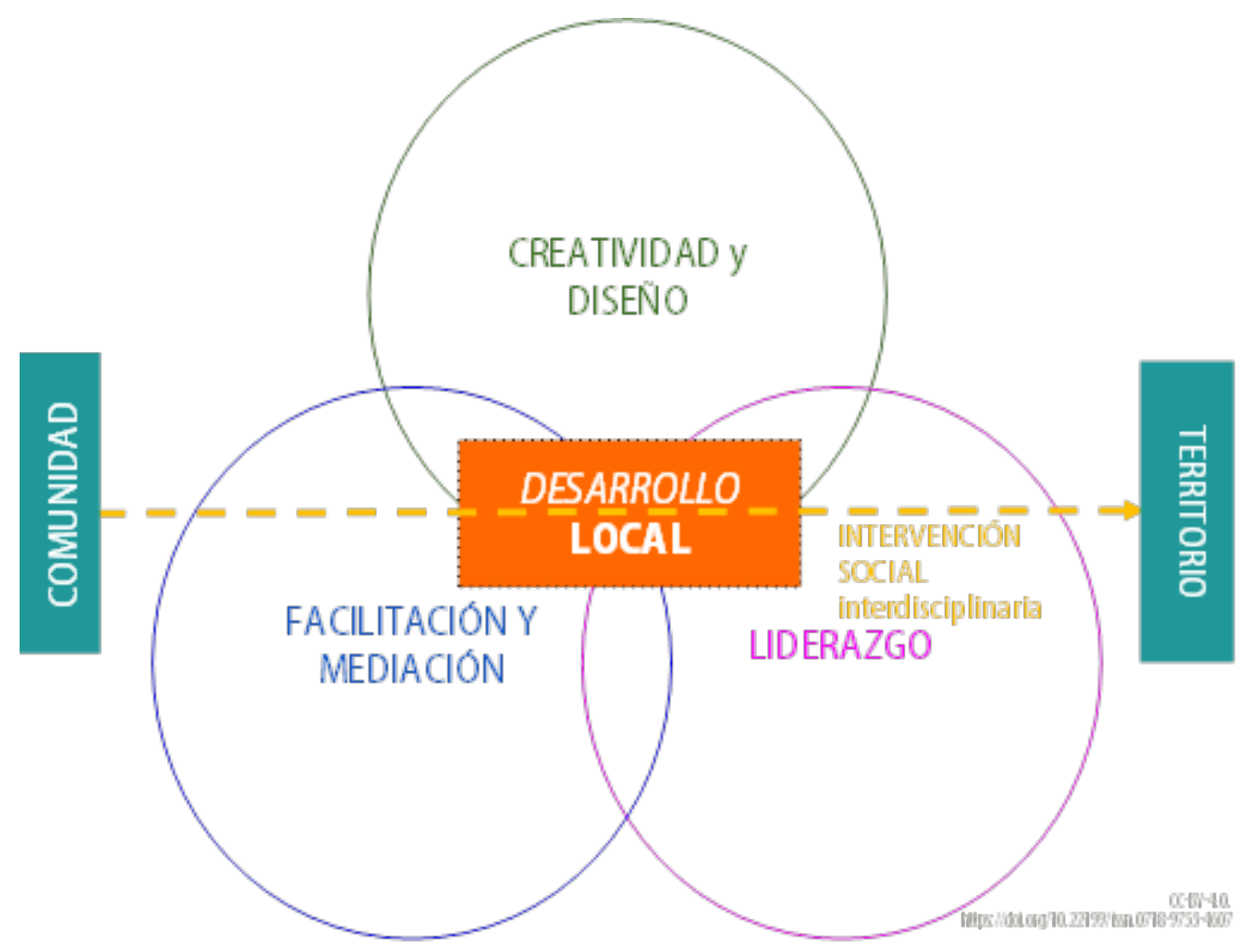

Figura 1: Enfoque de Innovación Social Territorial. (Elaboración propia).

Como se puede apreciar, la Figura 1 tiene como objetivo central de intervención al desarrollo local, el cual articula dos polos que se retroalimentan de manera permanente: comunidad y territorio, en tanto fuentes de creación de activos, recursos, relaciones y vínculos sociales endógenos. El proceso de innovación social otorga énfasis al co-diseño como estrategia de trabajo comunitario; entretanto, la perspectiva interdisciplinaria es indispensable, puesto que el desarrollo local es una construcción social multidimensional. Además, este diagrama presenta la facilitación y mediación (Freire, 1965) como elementos centrales para la formación de diversos actores sociales, involucrando sus propias experiencias, trayectorias y conocimientos. En este marco, el facilitador y mediador opera promoviendo el diálogo, organizando el conocimiento disponible y propiciando espacios creativos. Para este proceso se requiere de algún actor que ejerza el rol de activador o motor de transformaciones a nivel local. Por este motivo, resulta indispensable que los líderes sociales desarrollen competencias en este paradigma. A continuación se describen los principales componentes de este enfoque.

\section{Intervención Social Interdisciplinaria}

Los problemas sociales tienen un carácter multidimensional, lo que supone el desafío de abordarlos desde distintos dispositivos interdisciplinarios. La interdisciplinariedad, en este contexto, se comprende como una interacción entre dos o más disciplinas, organizadas para trabajar en conjunto sobre un 
problema común. Considera desde una simple comunicación, hasta la integración de conceptos, elaboración de metodologías, procedimientos e incluso epistemologías.

En este escenario, la discusión fundamental se puede plantear a partir de las siguientes preguntas: ¿Desde dónde intervenimos? ¿Qué ideologías, epistemologías, marco teóricos y metodologías sustentan las intervenciones sociales? Estos interrogantes facilitan encontrar contrapuntos o asociaciones armoniosas que permitan observar e intervenir en lo social de forma compleja e integral. Este diálogo interdisciplinar requiere generar de forma explícita espacios de intercambio, desplegar articulaciones discursivas complejas en coherencia con los fenómenos sociales que se intervienen (Salas, 2003, como se citó en Muñoz, 2011), aspecto que es clave en el enfoque de investigación-acción, puesto que el co-diseño de proyectos de innovación social se basa en una multiplicidad de voces, racionalidades e intereses.

En este sentido, una intervención social es un soporte para la construcción de sujetos diversos y autónomos en pro de un proyecto, incorporando las condiciones económicas, sociales y culturales en las que habitan. Las intervenciones sociales implican no sólo que cada actor haga lo que le corresponde, o que los distintos actores se organicen para evitar la redundancia de las acciones, sino que requiere, fundamentalmente, que los actores, desde su particularidad, se pongan de acuerdo (Cunill-Grau, 2014; Rojas y Haye, 2012). Se suma, entonces, la necesidad de una apertura y disposición a conocer otras estructuras cognitivas disciplinares, así como la capacidad de auto-observarse de manera constante, en estado vigilante, o bien como agentes sociales activos desde lo epistemológico, ideológico y conductual.

Desde este lugar y discusión, se fueron gestando las intervenciones sociales realizadas en la Escuela de Dirigentes Sociales, comprendiendo las problemáticas sociales de cada uno de los barrios de Santiago.

\section{Desarrollo Local y Territorio}

El desarrollo local es una perspectiva de transformación social, de carácter territorial, cuyo eje se encuentra en que sean las propias comunidades locales las que definan su perspectiva de futuro y el camino para llegar a este último. Con este fin, se propone reconocer y poner en valor un conjunto de elementos locales invisibilizados por el desarrollo de carácter nacional, generalmente centrado en variables económicas (Boisier, 2010). Así entonces, elementos como el capital social, el patrimonio, los bienes naturales, las capacidades y autonomías locales con respecto a centros político-administrativos, serán activos indispensables. Furtado (1982) señala que:

La experiencia ha demostrado ampliamente que el verdadero desarrollo es principalmente un proceso de activación y canalización de fuerzas sociales, de avance en la capacidad asociativa, de ejercicio de la iniciativa y de la inventiva. Por lo tanto, se trata de un proceso social y cultural, y sólo secundariamente económico. Se produce el desarrollo cuando en la sociedad se manifiesta una energía, capaz de canalizar, de forma 
convergente, fuerzas que estaban latentes o dispersas. (Furtado, 1982, p. 149, como se citó en Boisier, 2010, p. 13).

Se puede apreciar, por tanto, que el territorio no es solo un espacio neutro en donde habitan las comunidades (ver eje horizontal de la Figura 1), sino que se transforma en un elemento central, complejo y dinámico del desarrollo. Si bien el territorio vinculado al desarrollo local ha sido un concepto utilizado desde los años setenta (Capel, 2016), su profundización y uso es relevante debido a que es un elemento articulador fundamental, tanto desde el punto de vista teórico como empírico. En este sentido, se puede apreciar que los modos de vida, las experiencias cotidianas, los imaginarios sociales y los espacios significativos y normados de las personas ocurren precisamente en los territorios (Lindon, 2007; Ther, 2012), convirtiéndose en la fuente principal de conocimiento y recursos para la innovación social. No obstante lo anterior, estos recursos generalmente se articulan de manera tal que conservan el statu quo, motivo por el cual requieren de un insumo que no poseen, en general, otras intervenciones sociales, pero que resulta fundamental en el marco de la innovación social: la creatividad.

\section{Creatividad y Diseño}

La creatividad en innovación social ha sido uno de los fenómenos más explorados por este tipo de intervenciones, siendo la investigación en creatividad social y los procesos de desarrollo local y regional algunos de los más destacados (Moulaert et al., 2010), por lo que resulta aquí relevante detenerse en la capacidad de crear. En términos formativos o educacionales, esta capacidad es una de las más complejas del pensamiento (Bloom, Engelhart, Furst, Hill y Krathwohl, 1956), ocupando el nivel superior de aprendizaje. Se considera como un modelo metodológico que va más allá de la memorización y la comprensión del contenido específico que se trabaja en clase. Esta aproximación busca que los estudiantes/dirigentes lleguen a desarrollar habilidades que les permitan utilizar el conocimiento adquirido como una herramienta para resolver problemas y crear conocimiento a nivel cotidiano, en sus propias actividades y en función de múltiples desafíos diarios.

El diseño, entendido como una disciplina que desarrolla pensamiento creativo a través de técnicas visuales, maquetas y prototipos que permiten plasmar ideas y conceptos, será, en este contexto, un referente utilizado en la metodología ABP-NISA para estructurar procesos formativos que permitan lograr el aprendizaje por medio de experiencias prácticas e interactivas con el medio, el cual está compuesto por el grupo, la comunidad y el territorio en donde se realizan los cursos. Estimular la capacidad creativa en grupos de personas es posible mediante actividades y didácticas lúdicas que desarrollan habilidades colectivas por medio de herramientas especialmente diseñadas para facilitar procesos creativos, siendo uno de los momentos críticos la adaptación y validación territorial de las ideas, lo cual se realiza a partir de maquetas y prototipos. Espiau (2107) sostiene que:

Las iniciativas de innovación social deben pasar por un proceso de ajuste y adaptación al contexto local antes de ser implementadas. En lugar de plantear proyectos cerrados, las 
propuestas deben ser siempre sometidas a la opinión de los agentes locales para que puedan ser percibidas como propias y conectadas a las opiniones que se recogen en la fase de diagnóstico. (p. 147).

De manera complementaria, el design thinking es otro elemento metodológico central del proceso creativo. Se compone de cinco etapas: Empatizar, definir, idear, prototipar y testear (Brown, 2008), las cuales contribuyen a ordenar el trabajo de materialización de proyectos de manera flexible y creativa. En este caso, la metodología se complementa con otras formas de diseño centrado en las personas, tales como el conocimiento colectivo, la cooperación y la innovación abierta (Espiau, 2017, p. 147), con el fin de poder integrar las habilidades cognitivas de la creatividad con herramientas prácticas de diseño a escala local. Uno de los productos más relevantes de este proceso es el co-diseño de prototipos de innovación social, que sirven para materializar de manera rápida, lúdica y colaborativa las ideas de innovación. Según la Agencia de Innovación del Reino Unido:

La creación de prototipos es un enfoque para desarrollar, probar y mejorar ideas en una etapa temprana antes de que se comprometan recursos a gran escala para la implementación. Es una forma de proyecto y trabajo en equipo que le permite experimentar, evaluar, aprender, perfeccionar y adaptarse. Asegurarse de que las ideas se exploren por completo antes de sacar conclusiones. (Fox, 2015, p. 4).

\section{Liderazgo comunitario}

El liderazgo es un componente transversal en el proceso formativo de dirigentes sociales. Desde la mirada humanista-existencial y comunitaria a la cual adscribe la propuesta metodológica que aquí se presenta, es necesario comenzar por el ejercicio reflexivo de preguntarnos: ¿Qué se entiende por persona? ¿Desde dónde miramos a las personas con las cuales trabajamos e intervenimos? Siguiendo a Rogers (1961), la persona es un ser autorrealizable, siempre en un proceso de crecimiento, a partir de lo cual los individuos tienen la capacidad de experimentar y de saber cuáles son sus propios desajustes (Rage, 1996). Esto no quiere decir, sin embargo, que se desconoce el contexto político, social y cultural, pero sí se considera que cada persona tiene la posibilidad de decidir, crear, regular y potenciar su propio ser. Esta persona desarrolla a lo largo de sus experiencias una noción de sí mismo que en la psicología humanista se denomina Self o Yo, el cual se forma a partir de la interacción con el entorno, físico y social. Por lo tanto, se parte de la idea de que a la persona la motiva su autorrealización, teniendo la capacidad de crear y autorregularse, definiéndose a sí mismo o misma en torno a sus experiencias con otros y otras.

De este modo, se plantea que no existe algo así como un "líder", sino personas que, en interacción social permanente con otras personas, territorios, comunidades y organizaciones, se potencian y emergen como líderes. El liderazgo comunitario entiende al dirigente social como un líder entretejido con el ambiente y el territorio, el cual surge de las reuniones con su organización, o ante circunstancias que afectan a la comunidad y que implican actuar con urgencia o de manera planificada (Montero, 2006). Asimismo, se sostiene que el liderazgo es la fuerza motriz del desarrollo local y el canal a través del cual fluyen las características que permiten mejorar las capacidades de la comunidad 
para producir resultados mediante la utilización de los recursos de que dispone y a los que pone en valor, lo que se traduce en fortalecimiento comunitario (Montero, 2012). De aquí la importancia de usar metodologías que promuevan la conversación, diálogo, creatividad, construcción y negociación permanente (Martínez, 2011). Tal como señala Aguilar (2001), "la posibilidad de desarrollar la función social de participación, unida al ejercicio del liderazgo y el modo de vertebración del tejido social asociativo, condicionan las posibilidades de éxito de las experiencias comunitarias" (p. 87).

A continuación se presentan los resultados de la Escuela con el fin de visualizar de manera más clara la aplicación del modelo presentado hasta ahora.

\section{Resultados}

\section{La Escuela de Dirigentes Sociales (2018-2019)}

Se diseñó e implementó un proceso formativo de dirigentes sociales a partir del modelo de aprendizaje basado en el diseño de proyectos de innovación social (ABP-NISA), el cual fue aplicado a un territorio-barrio específico de la Comuna de Santiago, en el marco de la Escuela de Dirigentes Sociales llevada a cabo durante los años 2018 y 2019. Este modelo se basa principalmente en el método design thinking (Brown, 2008), sin embargo, ha sufrido una serie de modificaciones al incorporar elementos del aprendizaje basado en proyectos, el liderazgo comunitario y humanista, y, sobre todo, al definir el desarrollo local como finalidad del proceso.

La Escuela de Dirigentes Sociales, gestionada por la Ilustre Municipalidad de Santiago, seleccionó al equipo y el enfoque de NISA Consultores para diseñar e implementar los módulos de liderazgo, comunicación e innovación social, en los cuales participaron más de 162 dirigentes sociales de diversas organizaciones territoriales y funcionales, quienes fueron parte de 31 grupos de trabajo. El proceso de formación se organizó en tres niveles educativos: básico, medio y avanzado, compuestos por diversos módulos por nivel. Se implementaron más de 24 horas de capacitaciones a través de un proceso de trabajo continuo, durante el cual cada asistente debió incorporar las competencias requeridas en cada uno de los módulos a lo largo de los tres niveles.

Todos(as) los(as) dirigentes ingresan al nivel básico, en cursos de 25 a 30 personas, para luego continuar en los mismos grupos por los niveles medio y avanzado. Los requisitos de inscripción son residir en la comuna de Santiago y tener un cargo en alguna organización territorial o funcional de la misma comuna. De este modo, la Escuela se compone de una alta heterogeneidad social, cultural, económica, política y educacional. Los talleres fueron desarrollados en espacios tales como el Centro Cultural Palacio Álamos, auditorios municipales y espacios públicos. Las metodologías señaladas en la primera parte de este artículo se aplicaron de la siguiente manera: 
1) Adaptación del design thinking para la innovación social: Esta metodología, en su versión institucionalizada, usa la sensibilidad y el enfoque del diseño con el fin de hacer coincidir las necesidades de las personas (usuarios) con productos y servicios provenientes del mercado. Originalmente está orientada a la generación de nuevos productos y servicios a ser comercializados, para lo cual, como se ha mencionado, se transita por cinco etapas: empatizar, definir, idear, prototipar y testear. En términos generales, el proceso comienza con la comprensión del comportamiento y el entorno del usuario, con el fin de empatizar con él. Posteriormente, se definen problemas significativos para los usuarios, a partir de lo cual se idean múltiples soluciones. El siguiente paso, denominado prototipado, busca co-diseñar materialmente las mejores ideas seleccionadas con el fin de poder testear con los usuarios reales si el producto/servicio es valioso y útil. En la metodología ABP NISA, sin embargo, el design thinking es adaptado para la observación y resolución de problemáticas locales y comunitarias (ver Figura 2), fin con el cual se modifican las etapas del proceso de diseño original, facilitando procesos colectivos y heterogéneos.

En primer lugar, se ha incorporado la etapa "conectar" al inicio del proceso, en la que se busca que los asistentes estén conscientes tanto de sí mismos como del entorno (barrio) inmediato en donde se realiza el taller, lo cual se logra a través de técnicas de encuadre, toma de consciencia individual y observación etnográfica. La segunda modificación consiste en la profundización de la primera etapa del modelo original, denominada "empatizar", y se realiza al profundizar la observación del entorno, es decir, considerando la observación y la empatía como un proceso multidimensional, que permita dar sentido o hacer significativo el producto/servicio. Esta modificación ha incidido en que a la primera etapa del proceso se le denomine "observar" en lugar de empatizar (ver Figura 2). Una tercera y cuarta modificación consisten en incorporar las etapas "comunicar" y "financiar". La primera busca que los asistentes tengan las competencias para crear narrativas con propuestas de valor claras para sus grupos de interés; la segunda persigue la viabilidad financiera de los proyectos, tanto a través de la identificación de fondos públicos, como de la articulación público-privada, a través de modelos de responsabilidad social empresarial u otros similares.

2) Aprendizaje basado en proyectos: Se trata de una metodología activa, que busca aprendizajes cognitivos, colaborativos y de contenido (Kolmos, 2009), en la que los estudiantes se enfrentan a diversas situaciones similares a las que encontrarán en su vida cotidiana. Así entonces, los asistentes a la Escuela de Dirigentes deben co-diseñar, a través de una serie de pasos basados en el design thinking, tanto un problema relevante como una solución creativa, o una mejora, mediante el enfoque de innovación social aplicado a sus barrios.

Los enfoques metodológicos tienen objetivos claros y diferenciados: el objetivo del design thinking es co-diseñar productos o servicios centrados en la experiencia del usuario; el aprendizaje basado en proyectos, en tanto, tiene como finalidad generar procesos de aprendizaje por 
competencias, a través de la formulación de proyectos. Estas dos finalidades se integran en tanto la Escuela busca que el co-diseño de productos o servicios comunitarios se convierta en el proceso de aprendizaje a través del cual se desarrollan competencias específicas de innovación social. De este modo, las dinámicas de trabajo realizadas en cada una de las sesiones van entretejiendo elementos de cada una de las metodologías, fortaleciendo las competencias a partir de la participación en todos los niveles de aprendizaje. La Figura 2 señala los principales elementos y la secuencia de estas dos aproximaciones metodológicas:

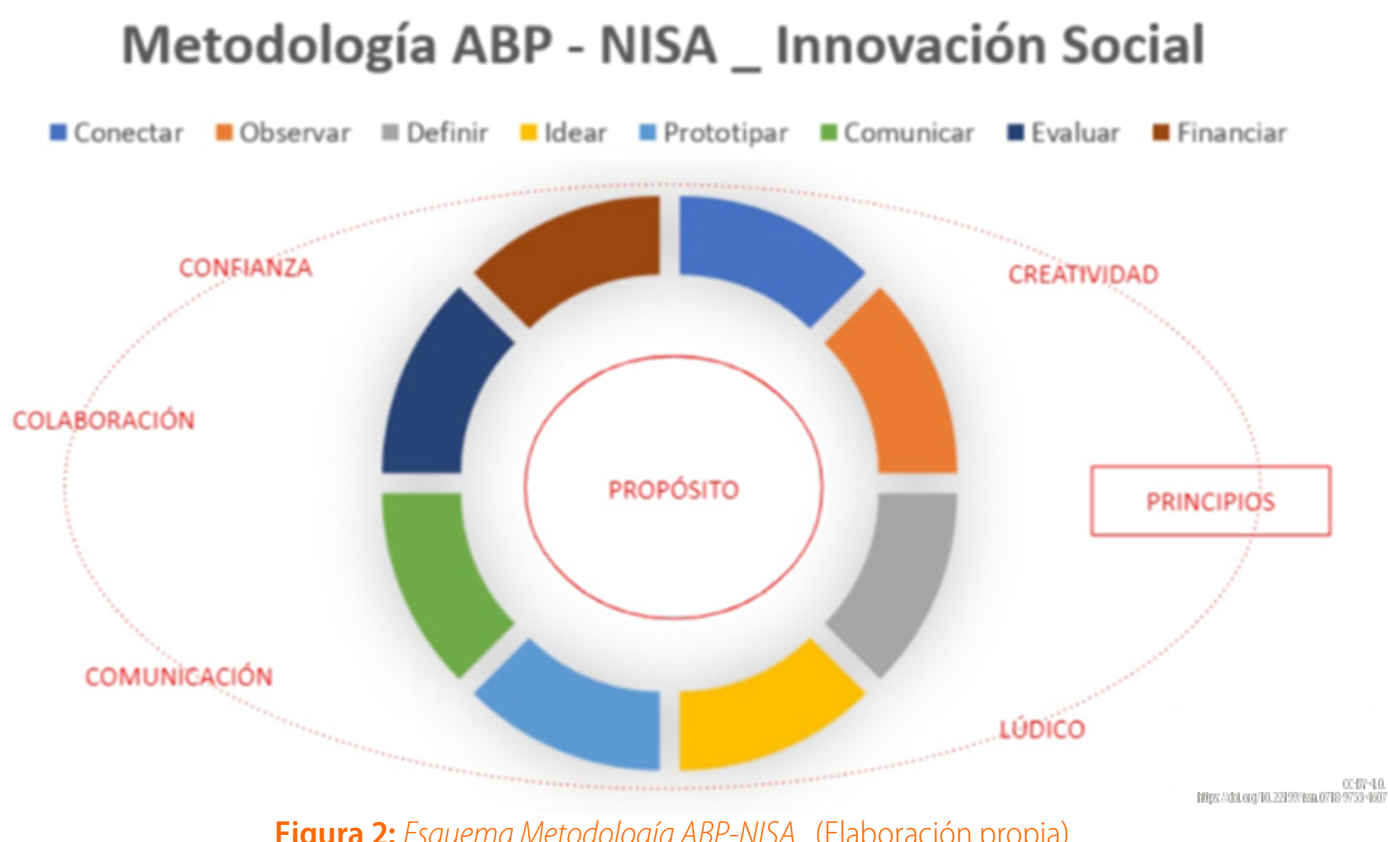

Figura 2: Esquema Metodología ABP-NISA. (Elaboración propia).

Como se puede apreciar, el proceso propiamente creativo de proyectos, que va desde el conectar hasta el financiar, se articula a una serie de elementos tales como la confianza, la colaboración, la comunicación, el espacio lúdico y la creatividad, que operan como principios que orientan el desarrollo de proyectos. De este modo, se busca integrar la experiencia que traen los dirigentes, situada, local y biográfica, con un propósito dirigido hacia el bien común. El tejido de este proceso, si cabe usar esta metáfora, son las metodologías creativas, las cuales abren un escenario de trabajo colectivo diferente al de los proyectos sociales tradicionales.

\section{Proceso de aprendizaje}

El nivel básico de la Escuela consistió en una introducción teórica a la innovación social como enfoque de intervención social. Se explicaron diversas aproximaciones, metodologías y tensiones éticas desde una mirada multidimensional del quehacer comunitario (social, político, económico, cultural, emocional, entre otros), y se complementó la formación con temáticas relacionadas: liderazgo comunitario y adaptativo, planteamiento de problemas y objetivos de intervención, y desarrollo 
creativo de intervenciones. Se emplearon tableros de pensamiento estratégico y lateral, ${ }^{1}$ así como diversas técnicas de mediación y facilitación de aprendizaje grupal (Freire, 1965). El objetivo de esta primera etapa era desarrollar la competencia de plantear problemas territoriales relevantes y visualizar escenarios potenciales de solución, y el producto final fue el co-diseño básico de un prototipo de innovación social.

En el nivel medio, se incorporaron aspectos territoriales a través de metodologías etnográficas y de la espacialización del territorio, mediante el trabajo cartográfico (mapeos participativos). ${ }^{2}$ En términos comunicacionales, se incorporó la presentación en público de los proyectos a través de la técnica elevator pitch o discurso del ascensor. ${ }^{3}$ En este nivel se buscó que los asistentes aprendieran a situar sus prototipos de innovación social en contextos socio-culturales y lograran comunicarlos a sus grupos de interés.

En el nivel avanzado, llamado Laboratorio de Innovación, se exploraron metodologías de trabajo grupal centradas en el testeo de proyectos innovadores, así como de su potencial financiamiento y escalabilidad. En la dimensión psico-social, se incorporan elementos del eneagrama (Naranjo, 1999), como una forma compleja de comprender el liderazgo desde su dinámica relacional. Como producto de esta etapa se buscaba determinar la factibilidad del prototipo de ser utilizado por el público de interés, así como estructurar un proyecto de innovación social a nivel territorial.

De acuerdo con lo observado en el proceso, cada uno de estos niveles permite ampliar el conocimiento, habilidades y competencias de los dirigentes según lo planteado en la Figura 2. En este sentido, destaca la articulación de metodologías creativas con las centradas en la planificación, configurando diversas aproximaciones cognitivas, emocionales y socio-culturales.

\section{Prototipos de innovación social}

Si bien los prototipos son co-diseñados colectivamente por los asistentes a la Escuela de Dirigentes Sociales en el nivel básico, se pueden considerar como los artefactos centrales del proceso completo, pues se trabaja en relación con ellos durante todos los niveles. En las versiones de la Escuela desarrolladas entre los años 2018 y 2019, entre estos prototipos destacaron iniciativas enfocadas en dinamizar la economía local a escala de barrio con perspectiva de género; la sensibilización ambiental por medio de acciones comunitarias festivas; la entrega de servicios de salud primarios con pertinencia cultural; y el mejoramiento urbano integrando el co-diseño de adultos mayores. Algunos de estos prototipos son los siguientes:

\footnotetext{
${ }^{1}$ Los autores de este artículo han desarrollado herramientas específicas en el marco de los servicios prestados a instituciones públicas, dentro de los cuales se encuentran diversas versiones de tableros de planificación, estrategia, entre otros.

2 Esta metodología está inspirada en el trabajo realizado por el equipo de investigación de la Universidad Católica de Valparaíso titulado "Territorios Alternativos".

${ }^{3}$ Esta técnica, de origen anglosajón, busca construir discursos o narrativas breves de alto valor para los grupos de interés del proyecto.
} 


\section{Combi Emprendedoras}

Consiste en un "local móvil" que opera como vitrina de productos y servicios entregados por una organización de mujeres emprendedoras de la comuna de Santiago, que no tiene recursos suficientes para pagar por el arriendo de un local. Se co-diseñó un modelo de gestión y administración del local móvil, para ordenar un recorrido por diversas plazas y espacios públicos de la Comuna, desarrollando una actividad de enganche con música y gastronomía, en coordinación con la Junta de Vecinos, a través de un calendario de programación que permita preparar a la comunidad para su llegada. La estrategia de financiamiento considera para la inversión inicial y gastos operativos el auspicio de empresas que apoyan el emprendimiento, así como el patrocinio municipal.

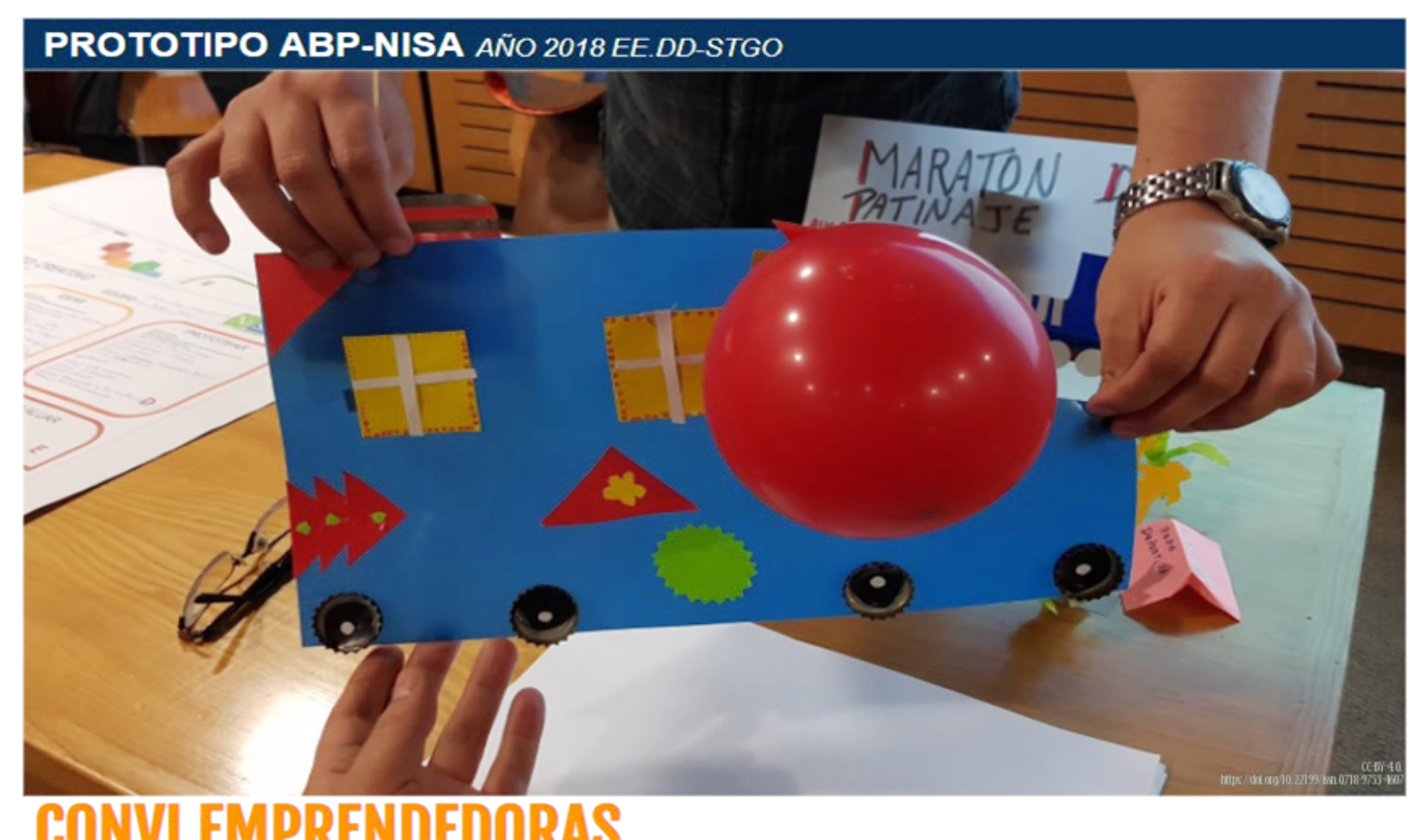

Figura 3: Prototipo Combi Emprendedoras. (Elaboración propia).

\section{Carnaval del Reciclaje}

Se trata de un evento a escala barrial con el objeto de sensibilizar y generar conciencia ambiental a partir de una actividad celebrativa -un carnaval-, que considera la construcción de carros alegóricos y confección de disfraces con material reciclado. La construcción de los carros y vestimenta se realiza a partir de un proceso comunitario articulado por una organización funcional en cooperación con la Junta de Vecinos, con quienes se definen al menos 3 alianzas organizadas por calles. Como estrategia de financiamiento se espera levantar el auspicio de empresas interesadas en promover la conciencia ambiental a nivel barrial, con el patrocinio de la municipalidad y la autogestión en el reciclaje de materiales por parte de la organización. Habrá diversos stands de programas y empresas sostenibles, y todo el material utilizado será dispuesto en container de reciclaje al terminar el evento, de manera de tener un bajo impacto ecológico. 


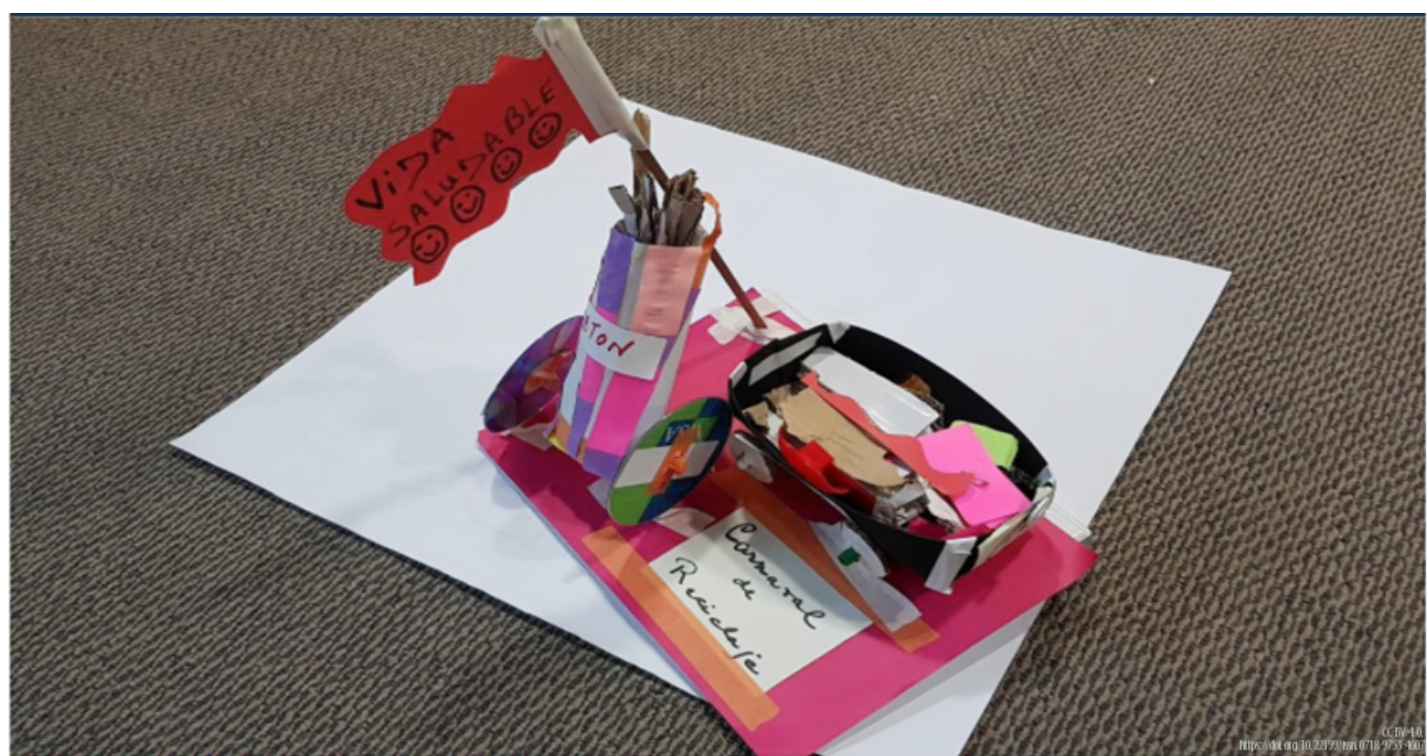

\section{CARNAVAL DEL RECICLAJE}

Figura 4: Prototipo Carnaval de Reciclaje. (Elaboración propia).

\section{Combi Salud Intercultural}

Se considera la adaptación de un vehículo tipo Combi para equipar un centro de atención de salud móvil, prestando servicios de carácter intercultural en diversas plazas de los distintos barrios de Santiago. El concepto guía de esta iniciativa será el Küme Mongen, palabra en mazupungún que significa buen vivir, y que es parte de la visión cultural de salud integral del pueblo mapuche. Entre la atención que se pretende brindar se incluye un chequeo general a los habitantes de los barrios, quienes serán convocados por medio de las redes de comunicación de la Junta de Vecinos y de la municipalidad, dándoles, además, la posibilidad de consultar por medicina complementaria con base en plantas. Como parte de la estrategia de funcionamiento se considera realizar un convenio con estudiantes en práctica de universidades de Santiago de las carreras de enfermería, medicina, kinesiología, salud bucal, entre otras similares, coordinados y coordinadas por la organización funcional a cargo de la operación del móvil. Para la inversión inicial se espera contar con apoyo financiero de la municipalidad o con recursos obtenidos mediante postulación a fondos concursables de carácter regional, y con auspicio para la operación por parte de empresas privadas interesadas en ser parte de la iniciativa dentro de su estrategia de Responsabilidad Social Empresarial (RSE). 


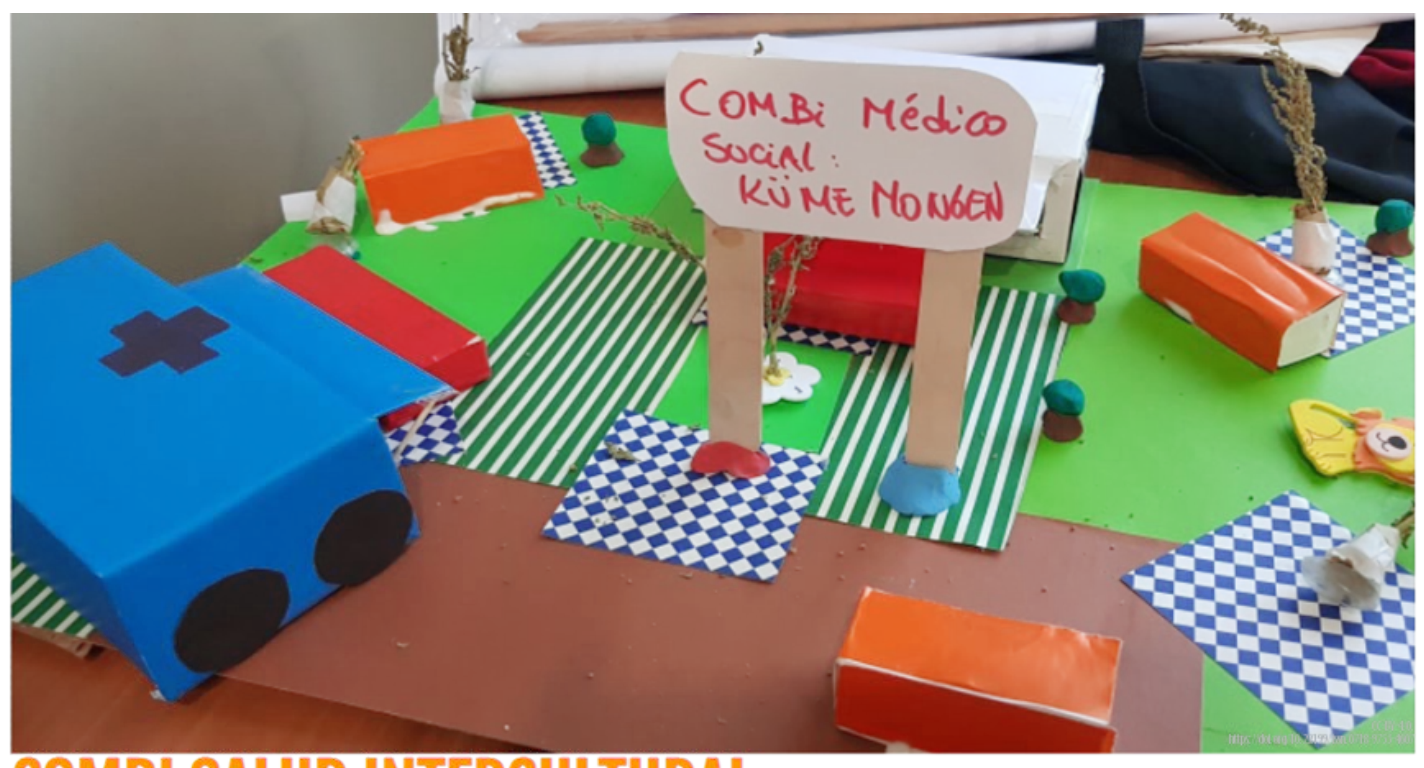

\section{COMBI SALUD INTERCULTURAL}

Figura 5: Prototipo Combi Salud Intercultural. (Elaboración propia).

\section{Plaza Integración para Adulto Mayor}

Este proyecto considera la renovación integral de una plaza ubicada en el sector céntrico de la ciudad, a partir de un proceso participativo de co-diseño con los y las adultos mayores del sector, quienes determinan el mobiliario, forma y equipamiento de la nueva plaza pensando en las necesidades de su grupo etario. Se identificó como problema la falta de ocupación de los espacios públicos, particularmente por parte de los adultos mayores, quienes han sido invisibilizados de los espacios existentes en el territorio. Como estrategia de financiamiento se plantea la formación de una agrupación funcional que sea capaz de postular a fondos concursables para el mejoramiento urbano, así como levantar fondos vía donación de empresas que sean vecinas del barrio, particularmente de las universidades que se emplazan en este lugar, las que además podrían participar del proceso comunitario de diseño y ejecución, ya sea con estudiantes o funcionarios. 


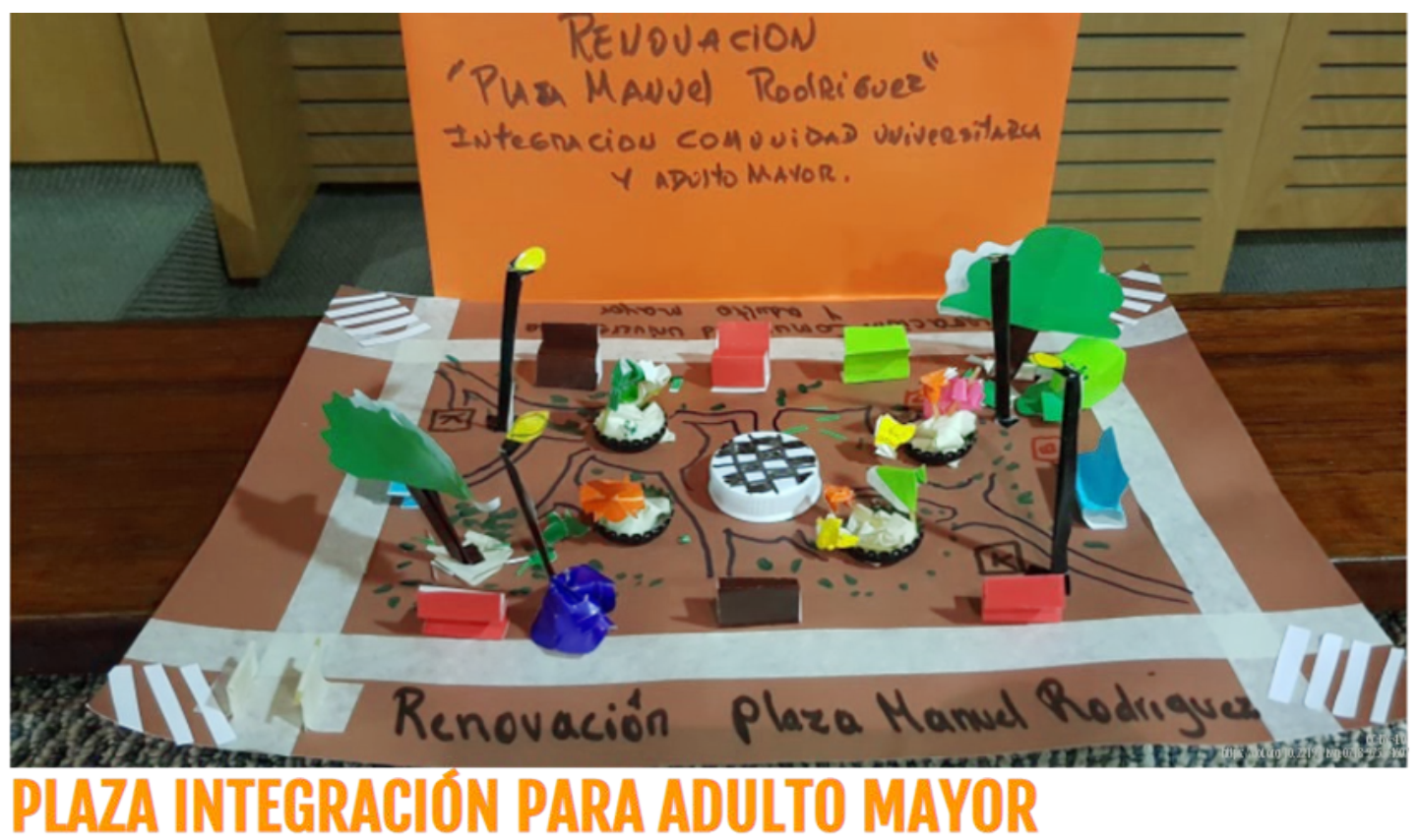

Figura 6: Prototipo Plaza Integración para Adulto Mayor. (Elaboración propia).

\section{Análisis e integración de resultados: de la activación del proceso de innovación social al desarrollo local}

El trabajo con los dirigentes sociales ha permitido apreciar que existe un proceso sinérgico entre la formación de líderes sociales, el co-diseño de propuestas de innovación social y el desarrollo local. Las extensas jornadas de trabajo con metodologías diversas, conversaciones y trabajo (formativo) en el barrio, permiten visualizar colectivamente la eventual incidencia de los proyectos de innovación social en el desarrollo local. En este sentido, se observa la necesidad de pensar los procesos de innovación social como intervenciones complejas y completas, más allá del rol que le corresponda a cada actor del ecosistema. La Figura 7 permite ilustrar de manera sintética, las etapas necesarias para un proceso de intervención completo, en el cual la Escuela de Dirigentes Sociales es apenas una parte.

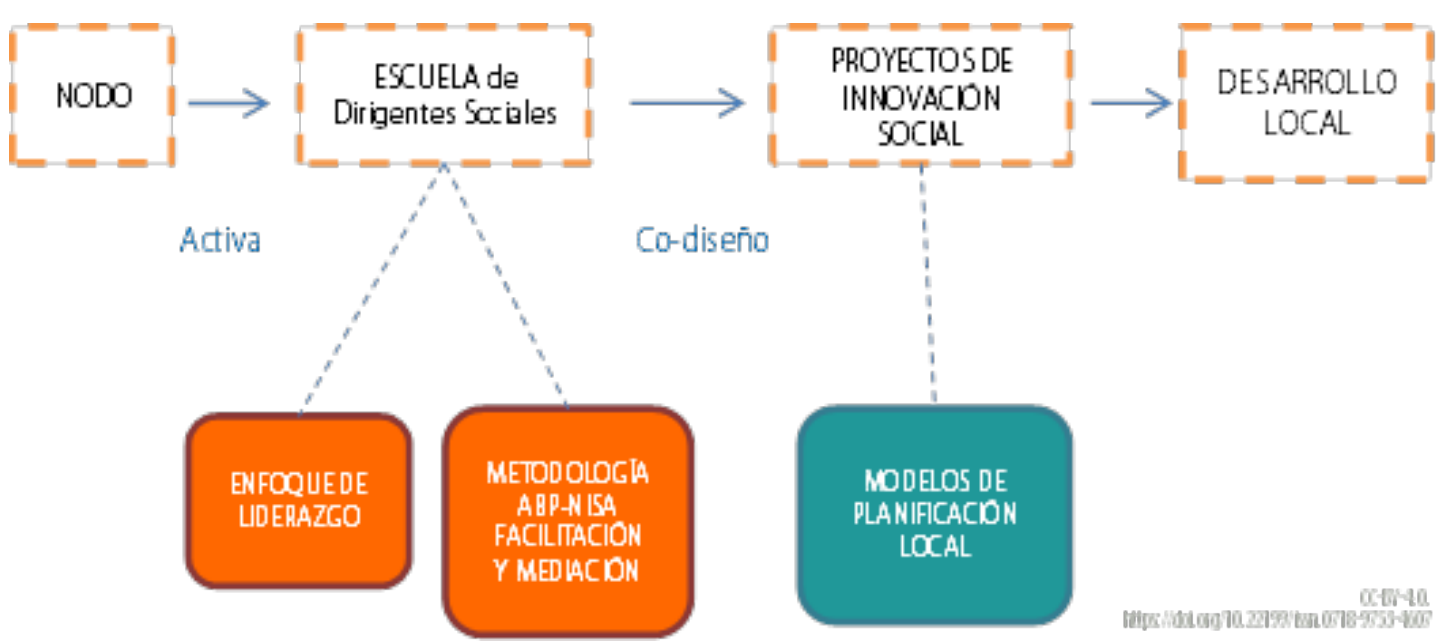

Figura 7: Proceso intervención social interdisciplinaria. (Elaboración propia). 
El proceso comienza con la activación de la intervención a través de un actor con capacidad de agencia en el ámbito de la innovación social. En este caso, el nodo es el municipio de Santiago, y el equipo de facilitadores especializados en innovación social y desarrollo local es NISA Consultores. La intervención, es decir, la Escuela de Dirigentes Sociales, contribuye a la formación de competencias en innovación social a través de dos líneas metodológicas: un enfoque particular de liderazgo, que combina elementos de la psicología humanista y comunitaria; y una perspectiva metodológica que articula el aprendizaje basado en proyectos, complementado con una adaptación local de la metodología design thinking, y fortalecido con un conjunto de prácticas educativas provenientes de las tradiciones de la facilitación y la mediación. A partir de estas metodologías, los dirigentes sociales co-diseñan prototipos y proyectos innovadores. Este aspecto es significativo, por cuanto no se trata de proyectos pre-existentes o de innovadores sociales que lleguen a capacitarse a la Escuela, sino que son dirigentes tradicionales que buscan aprender un camino diferente de transformación social.

Si bien la Escuela tiene como hilo conductor el co-diseño de prototipos de innovación social, el proceso requiere al menos dos pasos más para impactar en el desarrollo local. El primero es que el prototipo, una vez convertido en proyecto de innovación social, debe incorporarse a un modelo de planificación y gestión local que sea parte del paradigma de la innovación social, como puede ser el modelo multihélice, valor compartido, diálogo multi-actor, u otro; y no simplemente a los modelos actuales de intervención, puesto que, como se ha revisado a lo largo del artículo, no todo modelo de intervención es apropiado para un proyecto innovador. El segundo, es evaluar si ha logrado el impacto esperado en el desarrollo local, es decir, si la situación problemática inicial fue resuelta, al menos en alguna de sus dimensiones, por el proyecto de innovación social. La brecha entre el prototipo, o bien un proyecto de innovación social, y el impacto en el desarrollo local, está marcada por estos modelos de gestión, los cuales involucran a un ecosistema de actores comprometidos, o al menos vinculados, con este modo de pensar e intervenir el territorio.

\section{Conclusiones}

El presente artículo, situado en la tradición metodológica de la investigación-acción, ha buscado proponer reflexiva y críticamente un enfoque de intervención social interdisciplinario de formación de líderes sociales para el desarrollo local bajo el enfoque de la innovación social. Para ello se utilizó un estudio de caso único, la Escuela de Dirigentes Sociales de la comuna de Santiago, con el fin de ilustrar de manera empírica lo planteado.

Se realizó un análisis integrado del proceso llevado a cabo tanto en la Escuela como más allá de ella, con el propósito de contar con una mirada completa de una intervención social que considere desde la activación del proceso hasta su potencial impacto en el desarrollo local. En este contexto, la intención del artículo ha sido contribuir al debate académico sobre innovación social, al mismo tiempo que facilitar una discusión sobre enfoques, teorías y metodologías de intervención. 
De acuerdo con lo observado en el proceso de desarrollo de la Escuela de Dirigentes, la formación de líderes sociales en competencias de innovación social que apunten al desarrollo local puede contribuir a la generación de organizaciones, mecanismos y modelos de desarrollo más cooperativos, sustentables y creativos que los actuales, capaces de impulsar los cambios que requieren sociedades más democráticas y justas. Prueba de ello son los prototipos de innovación social codiseñados por los asistentes a la Escuela, puesto que reflejan una forma de trabajar dinámica, creativa y colectiva, diferente al paradigma con el cual llegan a este proceso formativo, caracterizado por una dirigencia social más bien individual, focalizada en proyectos sociales tradicionales financiados con fondos municipales, con grandes barreras de entrada para diseñar proyectos innovadores.

En este camino, la articulación conceptual y metodológica presentada, centrada en la combinación del aprendizaje basado en proyectos con una adaptación del design thinking, ha resultado ser particularmente útil para el trabajo con dirigentes sociales heterogéneos en su composición social, educacional y política.

Los resultados muestran que los dirigentes sociales desarrollan competencias en innovación social sin importar su origen socio-económico, adscripciones políticas o nivel educacional, siendo capaces de co-diseñar proyectos originales en conjunto con sus compañeros(as) de formación y los(as) facilitadores(as). De manera complementaria, al finalizar el proceso pueden diferenciar las estrategias individuales de innovación, centradas en la figura del emprendedor o "innovador genio/tecnológico", de aquellas aproximaciones colectivas que requieren amplios de espacios de cooperación y coordinación multi-actor.

En términos de sus resultados, sin embargo, el presente artículo no aborda los modelos de gestión local, la implementación, ni la evaluación de impacto de los proyectos de innovación social, por lo cual requiere sumarse a otras agendas de investigación, así como conocer iniciativas locales que estén en este proceso o que hayan pasado por él, con el fin de complementar y nutrir los resultados. El proceso completo es aún un asunto por estudiar tanto en términos teóricos como empíricos.

\section{Referencias}

Albuquerque, F. (2004). Desarrollo económico local y descentralización en América Latina. Revista de la Cepal, 82, 157-171. https://bit.ly/3ga9VBu

Aguilar, M. (2001). Participación comunitaria en salud: ¿Mito o realidad?. Díaz Santos.

Boisier, S. (2010). Descodificando el desarrollo del siglo XXI: subjetividad, complejidad, sinapsis, sinergía, recursividad, liderazgo, y anclaje territorial. Semestre Económico, 13(27), 11-37. https://bit.ly/2SoaKNO

Bloom, B. S. (Ed.).(1956). Taxonomy of educational objectives: The classification of educational goals. Cognitive domain (Vol. 1). Longmans. https://bit.ly/3xqMxFN 
Botella, A. y Ramos, P. (2019). Investigación-acción y aprendizaje basado en proyectos. Una revisión bibliográfica. Perfiles Educativos, 41(163), 127-141. https://doi.org/10.22201/iisue.24486167e. 2019.163.58923

Brown, T. (2008). Design Thinking. Harvard Business Review (Santiago. Edición en español), 86(9), 62-72.

Cahill, G. (2010). Primer on social innovation a compendium of definitions developed by organizations around the world. The Philanthropist, 23(3), 259-271. https://bit.ly/3wfh9JV

Capel, H. (2016). Las ciencias sociales y el estudio del territorio. Biblio3W. Revista Bibliográfica de Geografia y Ciencias Sociales, 21(1146), 1-38. https://bit.ly/3g86T0t

Colmenares, A. (2012). Investigación-acción participativa: una metodología integradora del conocimiento y la acción. Voces y silencios: Revista Latinoamericana de Educación, 3(1): 102-115. https://doi.org/10.18175/vys3.1.2012.07

Concha, R., y Ricci, E. (2018). Innovación Social Multihélice en la Región de Antofagasta. En E. Ricci y R. Concha (Eds.), Innovación Social. Consolidación Modelo Multihélice en la Región de Antofagasta (pp. 23-41). Antofagasta: Ediciones Universidad Católica del Norte. https://bit.ly/3zdNXFj

Córdoba-Cely, C., Villamarín, F., y Bonilla, H. (2014). Innovación social: aproximación a un marco teórico desde las disciplinas creativas del diseño y las ciencias sociales. Tendencias, 15(2), 30-44. https://doi.org/10.22267/rtend.141502.41

Cunill-Grau, N. (2014). La intersectorialidad en las nuevas políticas sociales. Un acercamiento analíticoconceptual. Gestión y Política Pública, 23(1), 5-46. https://bit.ly/356rnjN

Escobar, A. (2017). Diseño para las transiciones. Etnografías Contemporáneas, 3(4), 32-63. https://bit.ly/2Sq4pl5

Espiau, G. (2017). Nuevas tendencias en innovación social. Revista Española del Tercer Sector, 36(1), 141168. https://bit.ly/2SiQi16

Etzkowitz, H. y Leydesdorff, L. (1995). The Triple Helix -- University-Industry-Government Relations: A Laboratory for Knowledge Based Economic Development. EASST Review, 14 (1), 14-19. https://bit.ly/3zkKYef

Fox, D. (2015). Prototyping Framework. A guide to prototyping new ideas. Nesta, Thinkpublic. https://bit.ly/3v7XFpb

Freire, P. (1965). La educación como práctica de libertad. Siglo XXI.

Gatica, S. (2016). Innovación Social: Hacia una nueva aproximación del rol del Estado. Reflexiones. Consejo Nacional de Innovación para el Desarrollo. https://bit.ly/2TOnP3k

Hernández-Ascanio, J., Tirado-Valencia, P., y Ariza-Montes, A. (2016). El concepto de innovación social: ámbitos, definiciones y alcances teóricos. Revista de Economía Pública, Social y Cooperativa, 88, 164-199. https://bit.ly/3xhWBAP

Kolmos, A. (2009). Problem-Based and Project-Based Learning. En O. Skovsmose, P. Valero, y O.R. Christensen (Eds.), University Science and Mathematics Education in Transition (pp. 261-280). Springer. https://doi.org/10.1007/978-0-387-09829-6_13 
Lindón, A. (2007). Los imaginarios urbanos y el constructivismo geográfico: los hologramas espaciales. Eure (Santiago), 23(99), 31-46. https://doi.org/10.4067/S0250-71612007000200004

Malik, K. (2013). Informe sobre Desarrollo Humano 2013. El ascenso del sur: Progreso humano en un mundo diverso. Programa de las Naciones Unidas para el Desarrollo (PNUD). https://bit.ly/3cuUrFJ

Manzini, E. (2015). Design, when everybody designs. An introduction to design for social innovation. The MIT Press.

Martínez, A. (2011). Fortalecimiento comunitario desde el rol de las madres líderes: apuntes preliminares. Pensando Psicología, 7(12), 73-81. https://bit.ly/2TkjOmW

Mazzucato, M. (2014). El Estado emprendedor. Mitos del sector público frente al privado. RBA Libros S.A.

Ministerio de Desarrollo Social. (2018). Informe de Desarrollo Social 2017. https://bit.ly/3czaFxQ

Monnet, J. (2013). El territorio reticular (A. F. Cardona). En B. Nates, Enfoques y métodos en estudios territoriales (pp. 137-166). RETEC. (Obra original publicada en 2010). https://bit.ly/3iu6XcB

Montero, T. (2006). Teoría y práctica de la psicología comunitaria. La tensión entre comunidad y sociedad. Paidós.

Montero, M. (2012). El concepto de intervención social desde una perspectiva psicológica comunitaria. Patrimonio: Economía Cultural y Educación para la Paz (MEC-EDUPAZ), 1(1), 54-76. https://bit.ly/3pFIGTF

Moulaert, F., Swyngedouw, E., Martinelli, F., y Gonzalez, S. (Eds.). (2010). Can neighbourhoods save the city? Community development and social innovation. Routledge.

Muñoz, G. (2011). Contrapuntos Epistemológicos para intervenir en lo social ¿Cómo impulsar un diálogo interdisciplinar? Cinta Moebio, 40, 84-104. https://doi.org/10.4067/50717554X2011000100005

Naranjo, C. (1999). Autoconocimiento transformador. Los eneatipos en la vida, la literatura y la clínica. La Llave

Porter, M., y Kramer, M. (2011). La creación de valor compartido: cómo reinventar el capitalismo y liberar una oleada de innovación y crecimiento. Harvard Business Review (Santiago. Edición en español), 89(1), 31-49.

Prahalad, C.K. (2005). La riqueza en la base de la pirámide. Norma.

Rage, E. (1996). Ciclo Vital de la Pareja y la Familia. Universidad Iberoamericana.

Rodríguez, A. y Alvarado, H. (2008). Claves de la innovación social en América Latina. CEPAL. https://bit.ly/2RJ05wV

Rogers, C. (1961). El proceso de convertirse en persona. Paidós.

Rojas, C., y Haye, A. (Eds.). (2012). Actas Seminario Interdisciplina en la UC. https://bit.ly/3zieNvl

Rojas, R. (2013). El liderazgo comunitario y su importancia en la intervención comunitaria. Psicología para América Latina, 25, 57-76. https://bit.ly/3iFqeHU

Svampa, M. (2016). Debates latinoamericanos. Indianismo, desarrollo, dependencia y populismo. Edhasa. 
Ther, F. (2012). Antropología del Territorio. Polis (Santiago), 11(32), 493-510. https://doi.org/10.4067/S0718-65682012000200023

Yunus, M. (2006). ¿Es posible acabar con la pobreza? Complutense S.A.

\section{Para citar este artículo bajo APA 7}

Cortez López, M., Martínez Ríos, F. y Marcos Hernández, K. (2021). Formación de líderes sociales para el desarrollo local: una aproximación desde la innovación social. Social innovation studies, 01, e4607. https://doi.org/10.22199/ issn.0718-9753-4607

Copyright del articulo: @2021 Maurcio Cortez, Felipe Martínez y Kris Marcos 